EPJ Web of Conferences 19, 05001 (2012)

DOI: $10.1051 /$ epjconf/20121905001

(C) Owned by the authors, published by EDP Sciences, 2012

\title{
Observational constraints on the radial mixing of stars in the galactic disk
}

\author{
M. Haywood ${ }^{\mathrm{a}}$
}

GEPI, Observatoire de Paris, CNRS, Université Paris Diderot, 92190 Meudon, France

\begin{abstract}
Several mechanisms have been proposed in the recent years that could mix stars radially in the galactic disk, which include the effect of transient spiral arms, the interaction between the bar and the spiral structure, or the tidal interaction with a satellite. I review observational evidences of this radial mixing, discussing the properties of the solar vicinity metal-rich and metal poor stars, the possible differences in the kinematics of these two groups, and briefly comment on the evolution of the radial metallicity gradient.
\end{abstract}

\section{INTRODUCTION}

Several observational evidences show that stars in the young disk are born in an homogeneous ISM, with dispersion in abundances less than $0.05 \mathrm{dex}$, at a given place and time. Yet, samples of stars in the solar neighbourhood show dispersions in metallicities that are much higher, and it has long been suggested that this could reflect variations in the Galaxy rather than an evolutionary trend ([8] "...age may be less important than position in the Galaxy as a parameter of the abundance of disk population stars"). At least some stars observed locally could originate from the inner [6] and outer disk [1], which implies that some dynamical mechanisms operate in the disk that redistributing stars and bringing these objects to the solar vicinity. Several such mechanisms have been proposed in the recent years, that include transient spirals [21], resonance between the bar and spiral arms [14], or interaction(s) with satellite(s) [18]. In the following, we discuss possible observational indices that stars are radially mixed in the disk.

\section{WHY DO WE NEED MIXING?}

\subsection{Homogeneity of the ISM}

There are several evidences or indications that the young disk is and was chemically homogeneous now and in the past at a given galactic radius. B stars in the Orion star forming region show a high degree of homogeneity ([16], and references therein), with typical scatter of about 0.03 to 0.07 dex for various elements. [23] found 0.03 dex dispersion on measured iron abundances of a sample of 63 T-Tauri stars in 11 nearby associations. [4] found that the ISM within several hundred parsecs is very homogeneous, with dispersion below 0.04 dex within about $800 \mathrm{pc}$ for several species. Abundance ratios measured on solar neighbourhood stars of mixed ages (e.g [19]) have dispersions less than 0.04 dex, although the dispersion in metallicities is high. It must be emphasized that in all cases, dispersions essentially reflect measurement uncertainties, and are therefore upper limits. Finally, the analysis of isotopic ratios of various elements in presolar grains found in meteorites [17] shows that the cloud from which the

\footnotetext{
a e-mail: Misha.Haywood@obspm.fr
}

This is an Open Access article distributed under the terms of the Creative Commons Attribution-Noncommercial License 3.0, which permits unrestricted use, distribution, and reproduction in any noncommercial medium, provided the original work is properly cited. 


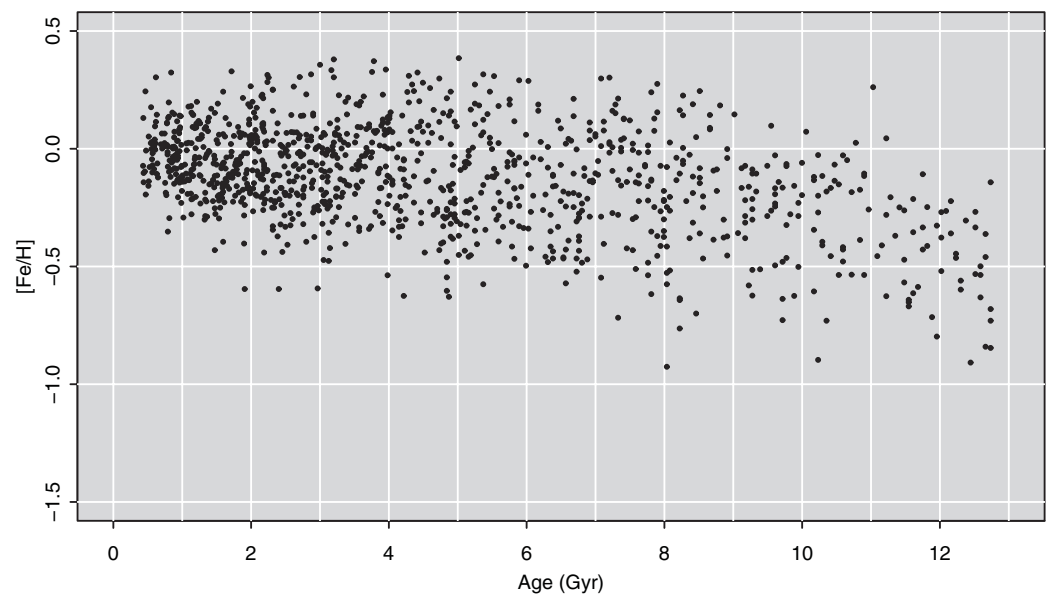

Figure 1. The age-metallicity distribution for a sample of stars in the solar neighbourhood. Metallicities comes from Geneva photometry, effective temperatures from V-K indices, and Hipparcos parallaxes from [22]. Ages using the methodology of [9].

solar system was formed was very well mixed, with dispersion possibly an order of magnitude below previously quoted limits.

\subsection{The age-metallicity distribution}

Although evidences exist that the interstellar medium from which stars are born is well mixed over scales of several hundreds of parsecs, the thin disk in the solar neighbourhood has metallicities in the range of -0.6 to +0.4 dex, with rms dispersion reaching $0.25-0.3$ dex for the oldest stars $([5,7])$. This is illustrated in Fig. 1, which shows a distribution of metallicities and ages for stars in the solar neighbourhood. Although recent determinations of the age-metallicity distribution have varied substantially in the last decade, and significant differences still exist between the various studies, there is an increasing consensus that (1) the mean metallicity has remained essentially constant in the last $10 \mathrm{Gyr}$, and (2) the dispersion increases with age, from values measured in the young disk to more than 0.2 dex. Such increase would be a natural consequence of radial mixing. Note that the Sun has often been cited as evidence that stars move from their initial orbits because of the measured difference between its metallicity and that of stars in the solar neighbourhood. It is however now found to be just $+0.05 \mathrm{dex}$ from the mean metallicity of stars of the same age.

\subsection{Mixing of stars}

Secular dynamical processes can mix the stars in the disk in different ways, for which [20] introduced the terminology of churning and blurring. Churning can change the mean orbital radius of the stars by several kpc, but with little effect on heating the orbits, which stay roughly circular. Blurring arises because stars are submitted irregularities of the gravitational potential and tend to diffuse with time. Stars oscillate radially with increasing larger amplitude, but keep the same mean orbital radius.

[21] and [3] derived estimates of the radial excursions of stars in the disk due to the increase of the epicycle amplitude of about $1.2 \mathrm{kpc}$ for a population with radial dispersion of $30 \mathrm{~km} / \mathrm{s}$. Over such distances, and in case of linear gradient of about $0.1 \mathrm{dex} / \mathrm{kpc}$, the range of expected metallicities in local samples would be of the order of $0.3 \mathrm{dex}$, which is much smaller than observed. Can the gradient be reasonably assumed to be linear? Observations are still fragmentary, but recent results seem to point to a rather flat distribution of metallicities beyond $\mathrm{R}_{G C}=9-10 \mathrm{kpc}$, at $[\mathrm{Fe} / \mathrm{H}]=(-0.3,-0.6)$ dex, a 
steep rise of metallicities towards the inner disk up to $[\mathrm{Fe} / \mathrm{H}]=(+0.0,+0.5)$ dex at about $\mathrm{R}_{G C}=6 \mathrm{kpc}$ (V. Hill, this conference), the mean metallicity then staying at this level towards the inner disk. It implies that stars with metallicities as low as $-0.6 \mathrm{dex}$ and as high as +0.5 dex are observed just $1-2 \mathrm{kpc}$ from the solar circle, not 5-6 kpc as it would be expected with a linear gradient of about $0.1 \mathrm{dex} / \mathrm{kpc}$. Stars in the tails of the MDF would then reach local samples due to their radial oscillations or with only limited churning. A question of interest is then if the observed step pattern of radial metallicity distribution is a consequence of radial mixing [10] or if it has been put in place as it is [24].

\section{EVIDENCES FROM LOCAL SAMPLES}

In case of strong mixing, the majority of old (age $>2$ Gyr) stars in the solar neighbourhood could have migrated from other radii [13]. Incidentally, it implies that we have to understand why the mean metallicity of stars that may have migrated over several kpc happens to be so near to the metallicity of the ISM at the solar radius. In any case, the a priori best candidates for migration in local samples are stars that occupy the extremes of the observed metallicity interval, the so-called super metal-rich stars $([\mathrm{Fe} / \mathrm{H}]>0.2 \mathrm{dex})$ and mild metal-poor stars $([\mathrm{Fe} / \mathrm{H}]<-0.3 \mathrm{dex})$.

\subsection{Metal-poor and metal-rich stars in the solar neighbourhood}

Star forming regions and B stars in the solar vicinity show typical solar abundances [16]. Spectroscopic metallicities of young open clusters within $\pm 1 \mathrm{kpc}$ of the solar galactocentric distance $(8 \mathrm{kpc})$ (calculated over 11 clusters younger than $1 \mathrm{Gyr}$ ), show mean metallicity of $0.05 \pm 0.09$ dex with the most metal-rich cluster being Praesepe at $[\mathrm{Fe} / \mathrm{H}]=+0.27$ dex. Finally, searches for super metal-rich stars in nearby star forming regions remain unsuccessful (e.g [2]). Hence, evidences are accumulating that the young disk at the solar neighbourhood presently doesn't form stars more metal-rich than $[\mathrm{Fe} / \mathrm{H}]=(+0.2,+0.3)$, and that these objects (less than $5 \%$ of the stars in the solar vicinity) have formed at some other places, the inner disk being their most probable origin.

Figures 1 and 2 show that stars with such metallicities $([\mathrm{Fe} / \mathrm{H}]>0.25 \mathrm{dex})$ are mostly confined to an age interval that goes from about 3 to $8 \mathrm{Gyr}$. Interestingly, the most metal-poor thin disk objects apparently reach greater ages, which raises several questions about their origin. If they have migrated from the inner disk, then we must understand why they are found preferentially at their pericenter (see next section) in local samples, which is more likely to suggest an origin in the outer disk. If their origin is truly the outer disk, then it would be interesting to know how their old ages fit with the inside-out disk formation scenario. Finally, some attention should be paid on the more or less equivalent proportion of these two groups of stars, since, if the vast majority of migrators is coming from the inner disk, we should expect some asymmetry in the MDF.

\subsection{What kinematic signature?}

It is unclear what signature radial mixing should leave in the kinematics of stars in the solar neighbourhood, with most attention focused on a possible correlation between the rotation velocity and metallicity, but with opposite expectations $([11,15])$. In [11], it is proposed that such correlation arises as an effect of the epicycle amplitude that makes stars originating from the outer or inner disk visible in the solar neighbourhood, with the mean guiding center of these objects still reflecting their origin. The correlation is expected to be seen on young ( $<2 \mathrm{Gyr})$ objects only, since churning of orbits would mix the stars of these different provenances and erase this signature.

Figure 3(a) shows the Vrot-[Fe/H] correlation found by [11] on young stars (age $<2 \mathrm{Gyr}$ ), as can be derived from the newest version of the GCS catalogue [5] (squares). But in fact a similar correlation is found in the age interval 4-15 Gyr, even though the inclusion of metal-poor thick disk stars lagging the LSR, probably weakens the correlation (triangles). Selection of stars in the age range $4<$ Age $<6$ Gyr 

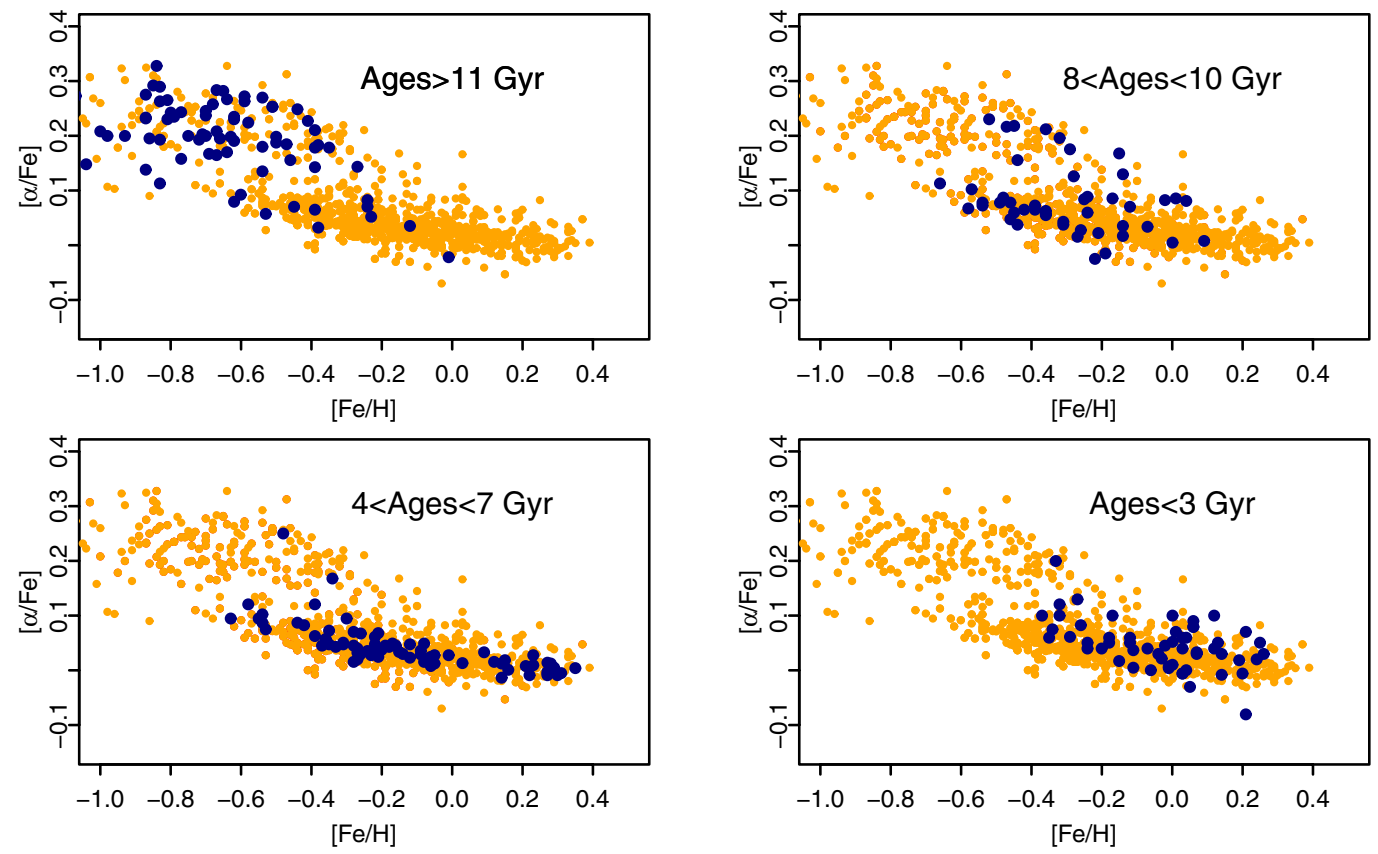

Figure 2. $[\alpha / \mathrm{Fe}]-[\mathrm{Fe} / \mathrm{H}]$ plot for a sample of solar neighbourhood stars with $\alpha$-element abundance ratios. In blue, stars for which the age could be determined. Note that (1) metal-poor thin disk stars reach greater ages than metalrich ones, and (2), metal-rich stars have ages in the range 3-8 Gyr.

(Fig. 3(b), same result is found between $6<$ Age $<8 \mathrm{Gyr}$ ), shows that a stronger correlation is found for older stars, contrarily to the predictions of [11]. Note that, most probably, the age upper limit at 6 Gyr is not sufficient to remove all thick disk stars: an even stronger correlation would probably be found if thick disk stars could properly be removed from the selection (Fig. 3(b), triangles).

Fig. 3(c) shows the (Rp, Ra) distribution of the 175 most metal-poor stars and similar number for the most metal-rich objects younger than 2 Gyr in the newest GCS catalogue. Fig. 3(d) shows the same for the $250 \times 2$ objects similarly selected in the age range 4-8 Gyr. Young metal-rich and metal-poor stars share similar distribution (not unexpectedly), and don't show the distinct pattern expected by [11]. On the contrary, old stars show markedly different behavior, with a large number of metal-poor stars reaching the solar circle at their pericenter, while most metal-rich objects reach their apocenter within $9 \mathrm{kpc}$, seemingly in contradiction with expectations of a well mixed old disk.

Finally, [15] assume such correlation should be indicative of radial mixing, since stars in the metalpoor tail of the thin disk that formed further out in the disk could have migrated to the solar circle loosing angular momentum, with the reverse applying to stars coming from the inner Galaxy [7]. It would then be expected that these two populations could have preserved some difference in angular momentum, which could therefore be seen in local samples. [15] found no correlation in their spectroscopic sample of solar neighbourhood stars. This is however also a consequence of the inclusion of thick disk stars in the selection, with the cut in $\alpha$-element ratios adopted to separate the thin and thick disks introducing stars with hot kinematics and significant lag, artificially lowering the mean rotation velocity of low metallicity disk stars. These stars do rotate faster than the metal-rich disk in the solar neighbourhood [7]. Whether this is a signpost of churning remains to be clarified.

\section{SIMPLE CONSIDERATIONS ON THE EVOLUTION OF GRADIENTS}

In principle, substantial mixing could modify, or even erase, pre-existing gradients. Depending on when exactly such mixing has occured, and on the possibility to rebuilt gradients from subsequent star 

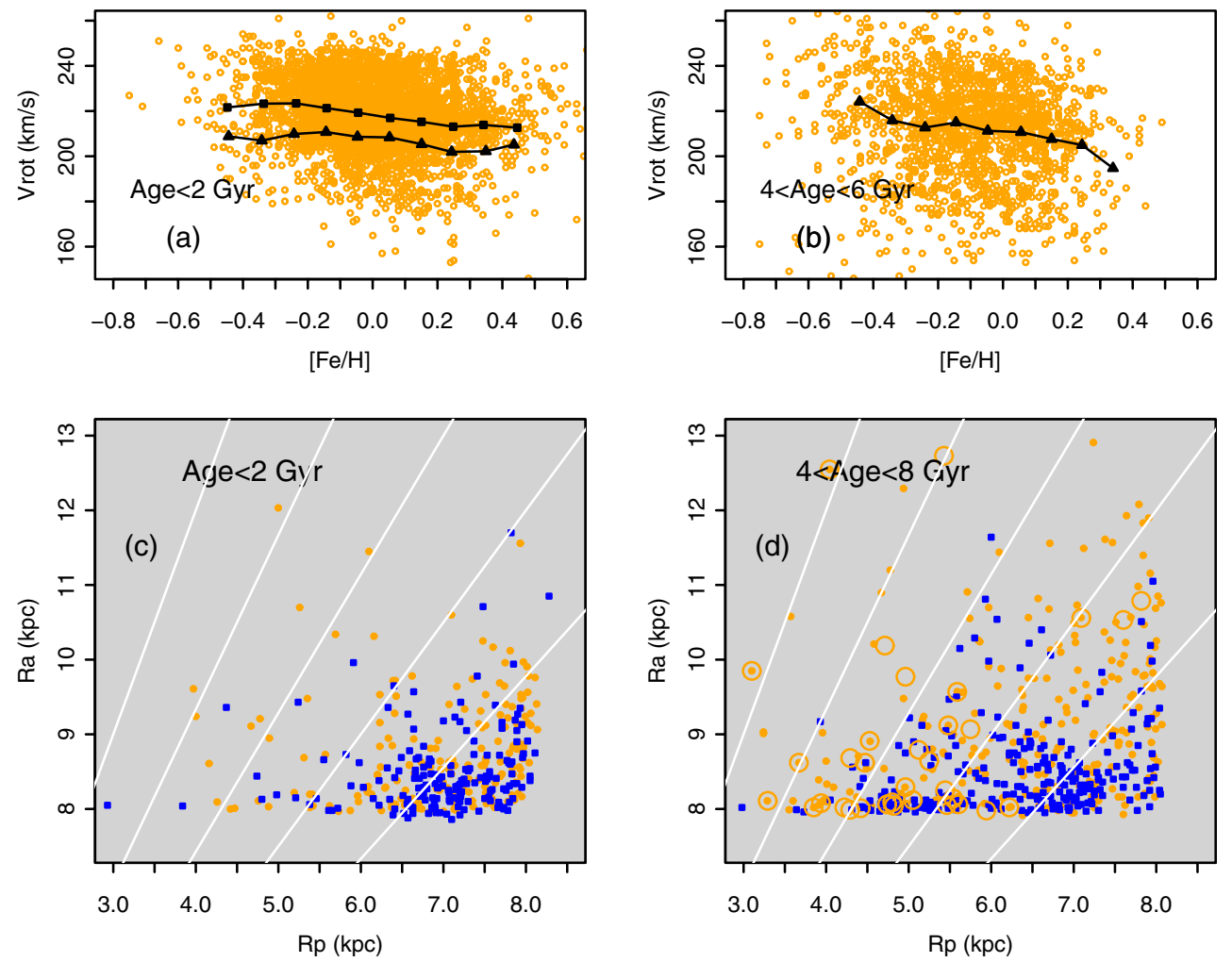

Figure 3. (a) Vrot-[Fe/H] distribution of stars younger than $2 \mathrm{Gyr}$ in the newest version of the GCS [5]. The mean velocity (squares) shows the (weak) trend mentioned by [11]. But the trend for older stars in the age range $4-15 \mathrm{Gyr}$ is similar (triangles). (b) Selection of stars in the range $4<$ Age $<6 \mathrm{Gyr}$ (mean relation, triangles), hence excluding part of the thick disk population. The correlation is stronger than the one observed for young stars. [11] suggested that young metal-rich or metal-poor stars, not yet submitted to radial mixing, would show systematic differences in (Rp, Ra) distribution, while old stars should not. On the contrary, it is observed that young metal-rich (blue squares) and metal-poor (orange circles) stars have similar distributions (c), which are markedly different for older stars (d). Straight lines are locii of constant eccentricity. Large circles are stars identified as thick disk objects according to their high level of $\alpha$-element abundance ratio.

formation activity and/or gas accretion, it may be expected to observe an increase of gradient with time. On the contrary, any substantial gradient in the old disk would suggest that mixing has only marginally redistributed the initial radial ordering of metallicities. Recent literature on chemical evolution has often emphasized evidences of a flattening of gradients, mainly based on the strong gradient of about $0.15 \mathrm{dex} / \mathrm{kpc} 8 \mathrm{Gyr}$ ago claimed by [12]. Given the metallicity of the solar neighbourhood 8 Gyr ago $([\mathrm{Fe} / \mathrm{H}]=-0.2 /-0.1 \mathrm{dex}$, Fig. 1$)$, it implies that the mean metallicity of the ISM at $4 \mathrm{kpc}$ in the inner disk would have already reached metallicities greater than 0.4 dex at that epoch. Even moderate mixing would have brought these stars to the solar circle, but they seem to be very rare or even nonexistent.

In case of strong mixing, any such correlation would be difficult to measure, because mixing would erase the initial ordering of the disk.

\section{CONCLUSIONS}

Studies of radial mixing in the Milky Way disk are still in their infancy, with almost every aspects needed to be investigated: the history and importance of mixing and its possible links with the barred episodes, relative importance of blurring and churning, etc. The possible signatures of these processes 
and the corresponding observational constraints remain largely unexplored. The available fragmentary constraints suggest that mixing has occurred, but it is unclear if it is a dominant process that has reshaped the structure of the disk or affected only a marginal number of stars.

The author thank Paola Di Matteo for several helpful comments and suggestions.

\section{References}

[1] Arp H., 1962, ApJ, 136, 66

[2] Biazzo K., Randich S., Palla F., Briceño C., 2011, A\&A, 530, A19

[3] Binney J., 2007, iuse.book, 67

[4] Cartledge S. I. B., Lauroesch J. T., Meyer D. M., Sofia U. J., 2006, ApJ, 641, 327

[5] Casagrande L., Schönrich R., Asplund M., Cassisi S., Ramírez I., Meléndez J., Bensby T., Feltzing S., 2011, A\&A, 530, A138

[6] Grenon M., 1972, ade..coll, 55

[7] Haywood M., 2008, MNRAS, 388, 1175

[8] Janes K. A., McClure R. D., 1972, BAAS, 4, 241

[9] Jørgensen B. R., Lindegren L., 2005, A\&A, 436, 127

[10] Lépine J. R. D., et al., 2011, MNRAS, 417, 698

[11] Loebman S. R., Roškar R., Debattista V. P., Ivezić Ž., Quinn T. R., Wadsley J., 2011, ApJ, 737, 8

[12] Maciel W. J., Costa R. D. D., 2009, IAUS, 254, 38P

[13] Minchev I., Famaey B., Combes F., Di Matteo P., Mouhcine M., Wozniak H., 2011, A\&A, 527, A147

[14] Minchev I., Famaey B., 2010, ApJ, 722, 112

[15] Navarro J. F., Abadi M. G., Venn K. A., Freeman K. C., Anguiano B., 2011, MNRAS, 412, 1203

[16] Nieva M.-F., Simón-Díaz S., 2011, A\&A, 532, A2

[17] Nittler L. R., 2005, ApJ, 618, 281

[18] Quillen A. C., Minchev I., Bland-Hawthorn J., Haywood M., 2009, MNRAS, 397, 1599

[19] Reddy B. E., Tomkin J., Lambert D. L., Allende Prieto C., 2003, MNRAS, 340, 304

[20] Schönrich R., Binney J., 2009, MNRAS, 396, 203

[21] Sellwood J. A., Binney J. J., 2002, MNRAS, 336, 785

[22] van Leeuwen F., 2007, A\&A, 474, 653

[23] Viana Almeida P., Santos N. C., Melo C., Ammler-von Eiff M., Torres C. A. O., Quast G. R., Gameiro J. F., Sterzik M., 2009, A\&A, 501, 965

[24] Vlajić M., Bland-Hawthorn J., Freeman K. C., 2009, ApJ, 697, 361 